Artigo Especial

Special Article

\title{
Consenso em Reumatologia Pediátrica Parte II - Definição de Melhora Clínica para o Lúpus Eritematoso Sistêmico e Dermatomiosite Juvenil(*)
}

\author{
Consensus in Pediatric Rheumatology \\ Part II - Definition of Clinical Improvement in Systemic Lupus \\ Erythematosus and Juvenile Dermatomyositis
}

\author{
Claudia Machado(1), Nicolino Ruperto ${ }^{(2)}$
}

\section{RESUMO}

Objetivo: avaliar as medidas de atividade e critérios de melhora clínica para o lúpus eritematoso sistêmico juvenil (LESJ) e dermatomiosite juvenil (DMJ), desenvolvidos por meio de consenso entre especialistas. Métodos e resultados: para o LESJ, as medidas essenciais em cinco domínios e as respectivas variáveis foram: 1) avaliação global pelo médico por escala analógica visual de 0-10 $\mathrm{cm}$; 2) avaliação da qualidade de vida relacionada à saúde (índice Físico-CHQ-PF50); 3) avaliação da atividade pelos pais/paciente por escala analógica visual de $0-10 \mathrm{~cm}$; 4) avaliação renal (proteinúria $24 \mathrm{~h}$ ); e 5) avaliação global da atividade por instrumento específico (SLEDAI ou ECLAM). A definição preliminar de melhora clínica para o JSLE foi: melhora $\geq 50 \% \mathrm{em}$ pelo menos 2 das 5 variáveis e não mais que uma com piora $>30 \%$, a qual não pode ser a proteinúria de $24 \mathrm{~h}$ em casos com envolvimento renal. Os seis domínios e as respectivas variáveis selecionadas para a atividade na DMJ foram: 1) avaliação global pelo médico por escala analógica visual de $0-10 \mathrm{~cm}$; 2) avaliação da força muscular proximal por meio de teste específico - CMASChildhood Myositis Assessment Scale 0-52; 3) avaliação da capacidade funcional (CHAQ); 4) avaliação da atividade pelos pais/ paciente por escala analógica visual de $0-10 \mathrm{~cm}$; 5) avaliação da qualidade de vida relacionada à saúde (Índice Físico - CHQ-PF50); 6) avaliação global da atividade por meio de instrumento específico (DAS - Disease Activity Score). A definição preliminar de melhora clínica para a DMJ foi: pelo menos 3 de quaisquer das 6 variáveis com melhora $\geq 20 \%$ e não mais que uma com piora $>30 \%$, a qual

\begin{abstract}
Objective: To evaluate the consensus development of the core response variables for disease activity in Juvenile Systemic Lupus Erythematosus (JSLE) and Juvenile Dermatomyositis (JDM) as well as the preliminary definition of improvement for both. Methods and Results: The core response domains and variables for JSLE were: 1) disease activity global assessment by the physician $=10 \mathrm{~cm}$ VAS; 2) health related to quality of life $=$ CHQ-PF50 physical score; 3) disease activity global assessment by parents $/$ patients $=10 \mathrm{~cm}$ VAS; 4) kidney assessment $=24 \mathrm{~h}$ proteinuria; 5) global disease activity assessment by a specific and valid tool (SLEDAI or ECLAM). The preliminary definition of improvement for JSLE was: 2 of the 5 measures with at least $50 \%$ of improvement and no more than 1 worse by more than 30\%, which cannot be the $24 \mathrm{~h}$ proteinuria. The core response domains and variables for JDM were: 1) disease activity global assessment by the physician $=10 \mathrm{~cm}$ VAS; 2) muscle strength assessment by a specific tool (CMAS - Childhood Myositis Assessment Scale) $=0-52$; 3) functional assessment by a valid tool (CHAQ); 4) disease activity global assessment by parents/patients $=10$ cm VAS; 5) health related to quality of life (CHQ-PF50 physical score); 6) global disease activity assessment by specific and valid tool (DAS - Disease Activity Score). The preliminary definition of improvement for JDM was: 3 of the 6 measures with at least $20 \%$ of improvement and no more than 1 worse by more than 30\%, which cannot be the muscle strength by
\end{abstract}

* Trabalho realizado na Disciplina de Reumatologia Pediátrica do Departamento de Pediatria da Faculdade de Medicina de Botucatu da Universidade Estadual Paulista (Unesp), Botucatu-SP, Brasil. Recebido em 22/07/2004. Aprovado, após revisão, em 09/12/2004.

1. Professora Assistente Doutora. Disciplina de Reumatologia Pediátrica do Departamento de Pediatria da Faculdade de Medicina de Botucatu da Unesp, Botucatu-SP, Brasil. Coordenadora e Representante Nacional do Paediatric Rheumatology International Trials Organization (Printo).

2. Senior Scientist. Paediatric Rheumatology International Trials Organization - (Printo) e Instituto G. Gaslini, Gênova, Itália.

Endereço para correspondência: Claudia Machado. Departamento de Pediatria. Faculdade de Medicina de Botucatu - Campus da Unesp, CEP 18618-970, Rubião Junior, Botucatu, SP, Brasil. Telefone: (14) 3811 6274 / (14) 3811-6083, fax: (14) 3882-0421. E-mail: cmachado@fmb.unesp.br 
não pode ser o CMAS. Conclusões: estas variáveis foram testadas em uma casuística representativa e mostraram propriedades estatísticas adequadas de responsividade e validade discriminativa, podendo ser estudadas em ensaios terapêuticos.

Palavras-chave: atividade da doença, consenso, dermatomiosite juvenil, lúpus eritematoso sistêmico juvenil.

\section{INTRODUÇÃO}

As doenças reumáticas pediátricas são consideradas doenças raras, pois têm incidência menor que 5-7,5/10.000. Entretanto, associam-se à morbidade elevada e grande impacto financeiro e social, causando incapacidade fisica e funcional, e afetando a qualidade de vida do paciente e de sua família. Incluem-se entre estas o lúpus eritematoso sistêmico juvenil (LESJ) e a dermatomiosite juvenil (DMJ).

Nas ultimas décadas houve um declínio importante na mortalidade causada pelo LESJ e pela DMJ, principalmente em razão da intervenção mais precoce e mais efetiva, com a introdução de protocolos terapêuticos com imunossupressores. Enquanto os ensaios clínicos controlados têm sido diligentemente conduzidos em adultos como forma de alcançar a melhor evidência, os ensaios terapêuticos em crianças e adolescentes com LESJ e DMJ são escassos. Entre os múltiplos obstáculos para a investigação destes pacientes estão: o pequeno número de casos em um único centro e uma definição de melhora que seja aceita internacionalmente.

Novas modalidades de tratamento e novos fármacos vêm sendo indicados no tratamento destas doenças, contudo a avaliação da resposta clínica ainda não foi padronizada. Novas perspectivas ainda estão por vir e requerem a avaliação por meio de ensaios clínicos controlados, para obter a melhor evidência tangível.

Múltiplas variáveis para definição do prognóstico e da melhora clínica do LESJ e da DMJ são relatadas na literatura $^{(1-7)}$, mas não existe ainda consenso sobre o que seja considerado melhora clinicamente significante. Conseqüentemente, os dados relativos à eficácia e segurança dos tratamentos para estas doenças provêm de estudos não controlados, com casuísticas pequenas e não comparáveis. Uma única definição de melhora clínica para o LESJ e para a DMJ facilitaria a padronização, condução e interpretação de ensaios clínicos controlados e ainda seria de grande valor na prática diária, na decisão sobre a melhora clínica resultante de diferentes modalidades terapêuticas. À longo prazo, melhoraria a eficiência de ensaios terapêuticos e de metaanálises.
CMAS. Conclusions: All the variables were tested and have shown good statistical responsiveness and discriminative validity to be used in therapeutic trials.

Keywords: consensus, disease activity, juvenile dermatomyositis, juvenile systemic lupus erythematosus.

Recentemente foram promulgadas por meio de consenso entre especialistas ${ }^{(8)}$ as medidas essenciais de desfecho clínico - "Core Set of outcome" para a "atividade" e "dano" tanto para o LESJ como para a DMJ ${ }^{(9,10)}$. São definidas como atividade todas as manifestações reversíveis que resultem diretamente do processo inflamatório. Define-se como danos causados pela doença, as alterações persistentes resultando em alterações anatômicas, fisiológicas ou patológicas em conseqüência da atividade inflamatória prévia, causando atrofia, cicatrizes ou fibrose, assim como as complicações do tratamento e outros eventos. As alterações relacionadas com os danos são freqüentemente irreversíveis e cumulativas, e/ou presentes por pelo menos seis meses. Diante da avaliação do dano cumulativo causado pelo lúpus em crianças e adolescentes, questiona-se também a validade dos instrumentos utilizados em adultos, pois o crescimento e desenvolvimento, assim como a capacidade regenerativa, peculiares à faixa etária pediátrica, precisam ser avaliados para assegurar a sua validade ${ }^{(9)}$.

As medidas consideradas essenciais para a atividade foram agrupadas em domínios e as variáveis que mensuram estes domínios foram selecionadas, como um número mínimo para avaliação a curto prazo da atividade da doença, visando a avaliar a resposta terapêutica na prática médica vigente e em futuros ensaios terapêuticos. Uma coleta de dados, prospectiva e em larga escala, obtida por meio de recrutamento internacional, foi realizada durante três anos entre 2001 e 2003, para a validação estatística destes critérios na prática.

\section{CRITÉRIOS DE INCLUSÃO}

Foram recrutados pacientes, após obtenção do consentimento livre e esclarecido dos pais ou representantes legais, que atenderam aos seguintes critérios:

1. Início recente e diagnóstico confirmado pelos critérios diagnósticos vigentes do LESJ ${ }^{(11,12)}$ ou DMJ ${ }^{(13)}$ e idade de início antes dos 18 anos.

2. Idade menor que 18 anos ao recrutamento.

3. Doença em atividade, tendo o paciente pelo menos um dos seguintes critérios: 1 . início recente de tratamento 
com corticosteróides e/ou imunossupressores; 2 . aumento da dosagem previamente utilizada de corticosteróides ou imunossupressores, ou introdução de nova modalidade de tratamento, devido à evidência clínica de atividade.

\section{CRITÉRIOS DE EXCLUSÃO}

Não foram considerados para este estudo os pacientes:

1. Em remissão espontânea ou induzida pelo tratamento que estivessem sem tratamento medicamentoso por pelo menos três meses.

2. Com atividade controlada e com doses estáveis de esteróides e imunossupressores por pelo menos três meses.

3. Com atividade considerada mínima pelo julgamento do médico.

4. Que apresentassem outras doenças graves associadas que pudessem interferir na atividade, evolução e tratamento.

O conjunto das variáveis essenciais foi registrado inicialmente durante períodos de atividade inflamatória e com intervalo de 6-8 meses a partir do recrutamento, em pacientes recebendo o tratamento adotado pelo médico responsável, de acordo com a rotina preconizada no hospital encarregado pelo seu atendimento.

\section{DEFINIÇÃO POR MEIO DO CONSENSO DE MELHORA CLÍNICA}

Durante a International II Consensus Conference on defining improvement in Juvenile Systemic Lupus Erythematosuis (JSLE) and Juvenile Dermatomyositis (JDM) Camogli Gênova, Itália, as variáveis selecionadas entre as medidas essenciais de desfecho clínico - Core set of outcome - foram testadas em pacientes reais, quanto à sua validade estatística. O perfil de 518 pacientes com LESJ e 284 com DMJ, provenientes de 45 países, foi analisado. É importante salientar a contribuição substancial para este projeto, de pacientes brasileiros com LESJ (63) e com DMJ (29), provenientes de centros universitários de Reumatologia Pediátrica* com a colaboração de membros associados ao Printo (citação em agradecimentos). A validade estatística do core set incluiu a estatística descritiva dos dados coletados incluindo os dados incompletos, a responsividade, ou seja, a variação em determinado intervalo de tempo, a validade discriminativa, ou seja, a capacidade de discriminar pacientes que tiveram melhora clínica dos que não apresentaram melhora, a validade de "construção" ou a comparação com o gold standard, a redundância e a consistência interna dos itens para avaliar a coesão das variáveis entre si. Mediante os resultados encontrados, em conjunto com a discussão ampla de forma organizada por meio da Nominal Group Technique( ${ }^{(8)}$, foram testadas as definições de melhora clínica para o LESJ.

\section{LUPUS ERITEMATOSO SISTÊMICO JUVENIL}

As variáveis previamente selecionadas, assim como a definição de melhora clínica, foram obtidas após análise de 128 perfis de pacientes, sendo então delineadas estatisticamente 597 definições possíveis. As definições do consenso foram aquelas que tiveram a concordância de pelo menos $80 \%$ dos participantes, em conjunto com o melhor teste de sensibilidade, especificidade e a área sob a curva da sensibilidade e especificidade combinadas.

\section{DOMÍNIOS E VARIÁVEIS SELECIONADAS PARA A ATIVIDADE NO LESJ}

1. Avaliação global da atividade pelo médico - escala analógica visual $-10 \mathrm{~cm}$.

2. Avaliação da qualidade de vida relacionada à saúde - Índice Físico do CHQ-PF5 $0{ }^{\circledR}$ Childhood Health Questionnaire ${ }^{(14)}$.

3. Avaliação do bem-estar global pelos pais/paciente escala analógica visual $-10 \mathrm{~cm}$.

4. Avaliação renal - Proteinúria de 24 horas.

5. Avaliação global da atividade por meio de instrumento específico - SLEDAI ${ }^{(1,15)}$ ou $\operatorname{ECLAM}^{(16)}$

* Parâmetros imunológicos - Título de anticorpos antiDNA - apenas descritivo.

* Determinação do índice psicossocial do CHQ-PF50 ${ }^{\circledR}-$ apenas descritivo.

\section{DEFINIÇÃO PRELIMINAR DE MELHORA CLÍNICA PARA O LESJ}

Duas de quaisquer das cinco variáveis com melhora de pelo menos $50 \%$ e não mais que uma com piora de mais de $30 \%$, a qual não pode ser a proteinúria de 24 horas em pacientes com envolvimento renal (a adição deste critério de restrição aumentou significantemente a especificidade da definição).

\section{DERMATOMIOSITE JUVENIL}

As variáveis selecionadas e a definição de melhora clínica foram obtidas após análise de 128 perfis de pacientes, sendo assim delineadas estatisticamente 992 definições possíveis, e as definições de consenso com a concordância de pelo menos $80 \%$ dos participantes, em conjunto com o melhor teste de sensibilidade, especificidade e a área sob a curva da sensibilidade e especificidade combinadas. 


\section{DOMÍNIOS E VARIÁVEIS SELECIONADAS PARA A ATIVIDADE NA DMJ}

1. Avaliação global pelo médico da atividade - escala analógica visual $-10 \mathrm{~cm}^{(17)}$.

2. Avaliação da força muscular proximal - CMAS Childhood Myositis Assessment Scale ${ }^{(18,19)}$.

3. Avaliação da capacidade funcional - Índice da Capacidade Funcional do CHAQ - Childhood Health Assessment Questionnaire ${ }^{(20)}$.

4. Avaliação do bem-estar global pelos pais/paciente escala analógica visual $-10 \mathrm{~cm}^{(17)}$.

5. Avaliação da qualidade de vida relacionada à saúde - Índice Físico do CHQ-PF50 ${ }^{\circledR}$ - Childhood Health Questionnaire ${ }^{(14)}$.

6. Avaliação global da atividade - DAS - Disease Activity Score ${ }^{(21,22)}$.

* Determinação dos valores séricos das enzimas musculares (creatina-fosfokinase-CPK, desidrogenase lática-DHL, aldolase, aspartato aminotransferase-AST, alanina aminotransferase-ALT) - apenas descritiva.

* Determinação do Índice Psico-Social do CHQ-PF50 apenas descritivo.

\section{DEFINIÇÃO PRELIMINAR DE MELHORA CLÍNICA PARA}

\section{A $D M J$}

Três de quaisquer das seis variáveis com melhora de pelo menos 20\% e não mais que uma com piora de mais de $30 \%$, a qual não pode ser o CMAS (a adição do critério de restrição aumenta a especificidade da definição).

Estes critérios foram selecionados independentemente dos critérios propostos para adultos com $\mathrm{LES}_{(23)}$ e também pelos critérios propostos por outro grupo de estudo - o International Myositis Assessment and Clinical Studies (IMACS) Group ${ }^{(24,25)}$ em adultos e crianças com dermatomiosite e os resultados alcançados para o critério de melhora clínica foram muito semelhantes.

Importante salientar que para ambas as definições de melhora clínica, no LESJ e DMJ foram incluídos domínios com avaliação da percepção da doença pelo paciente e por seus pais, assim como medidas válidas da qualidade de vida relacionada à saúde ${ }^{(26,27)}$, o que vem ao encontro das recomendações internacionais para a boa prática clínica, assim como de agências reguladoras de medicamentos para procedimentos em ensaios terapêuticos.

Participaram da II International Consensus Conference on Defining Improvement in Juvenile Systemic Lupus
Erythematosus (JSLE) and Juvenile Dermatomyositis (JDM)Camogli-Genoa-ITALY September 27-30, 2003(28), os seguintes membros:

\section{Organizadores:}

Alberto Martini, Genoa-Italy, Nicolino Ruperto, Gênova, Itália; Angelo Ravelli, Gênova, Itália; Ângela Pistorio, Gênova, Itália; Edward Giannini, Cincinatti, Ohio, EUA. Daniel Lovell, Cincinatti, Ohio, EUA; Boel Andersson-Gäre, Jönköping, Suécia.

\section{Membros:}

Paediatric Rheumatology International Trials Organization (PRINTO)

Carmen de Cunto, Buenos Aires, Argentina; Ruben Cuttica, Buenos Aires, Argentina; Rik Joos, Gent, Bélgica; Claudia Machado, Botucatu, Brasil; Sheila de Oliveira, Rio de Janeiro, Brasil; Dimitrina Myhaylova, Sofia, Bulgária; Miroslav Harjcek, Zagreb, Croácia; Pavla Dolezalova, Praga, República Tcheca; Susan Nielsen, Copenhagen, Dinamarca; Pekka Lahdene, Helsink, Finlândia; AnneMarie Prieur, Paris, França; Hans-Iko Huppertz, Bremen, Alemanha; Florence Kanakoudi Tsakalidou, Thessaloniki, Grécia; Yocef Uziel, Safed, Israel; Ingrida Rumba, RigaLatvia; Ruben Burgos-Vargas, Cidade do México, México; Nico Wulffraat, Utrecht, Holanda; Madeleine Rooney, Belfast, Irlanda do Norte, Berit Flato, Oslo, Noruega, Wierzbowska Malgorzata, Varsóvia, Polônia; Jose Antonio Melo-Gomes, Lisboa, Portugal; Irina Nikishina, Moscou, Rússia; Richard Vesely, Kosike, Eslováquia; Tadej Avcin, Ljubliana, Eslovênia; Michaël Hofer, Lausanne, Suíça; Huri Ozdogan, Istanbul, Turquia; Clarissa Pilkington, Londres, Reino Unido; Gordana Susic, Belgrade, Sérvia.

\section{Pediatric Rheumatology Collaborative Study Group (PRCSG)}

Brian Feldman, Toronto, Canadá; Lisa Rider, Bethesda, EUA; Philip Hashkes, Cleveland, EUA; Lauren Pachman, Chicago, EUA; Robert Rennebohm, Columbus, EUA; Anne Reed, Rochester, EUA; Carol Wallace, Seattle, EUA.

\section{External Observers}

Jenny Pratsidou, Thessaloniki, Grécia; Marcia Bandeira, Curitiba, Brasil; Stella Maris Garay, La Plata, Argentina.

\section{PATROCINADORES}

Paediatric Rheumatology International Trials Organization (PRINTO) - European Union - Contract n QLG1-CT2000-00514-EU. 
Pediatric Rheumatology Collaborative Study Group (PRCSG), NIH and Children's Hospital of Cincinnati Grant RO3 AI 44046-USA.

\section{AGRADECIMENTOS}

Aos colaboradores no recrutamento deste projeto, participaram os seguintes membros de centros brasileiros, afiliados ao PRINTO: Sheila Knupp Feitosa de Oliveira, Universidade Federal do Rio de Janeiro (UFRJ); Flavio

\section{REFERÊNCIAS}

1. Bombardier C, Gladman DD, Urowitz MB, Caron D, Chang CH: Derivation of the SLEDAI - a disease activity index for lupus patients. The committee on prognosis studies in Systemic Lupus Erythematosus. Arthritis Rheum 35:630-40,1992.

2. Gladman D, Ginzler E, Goldsmith C, et al: The development and initial validation of the Systemic Lupus International Collaborating Clinics/American College of Rheumatology Damage Index for Systemic Lupus Erythematosus. Arthritis Rheum 39: 363-9, 1996.

3. Isenberg D, Ramsey-Goldman R: Assessing patients with Lupus: towards a drug responder index. Rheumatology 38: 1045-9, 1999.

4. Strand V, Gladman D, Isenberg D, Petri M, Smolen J, Tugwell P: Outcome measures to be used in clinical trials in Systemic Lupus Erythematosus. J Rheumatol 26: 490-7, 1999.

5. Ward MM, Marx AS, Barry NN: Comparison of the validity and sensitivity to change of 5 activity indices in Systemic Lupus Erythematosus. J Rheumatol 27: 664-70, 2000.

6. Brunner HI, Silverman ED, To T, Bombardier C, Feldman B: Risk factors for damage in childhood onset of Systemic Lupus Erythematosus. Cumulative disease activity and medication use predict disease damage. Arthritis Rheum 46: 436-44, 2002.

7. Rider LG: Assessment of disease activity and its seqüelae in children and adults with myositis. Curr Opin Rheumatol 8:495-506, 1996.

8. Delbecq AL, Van De Ven AH, Gustafson DH (Ed): Group Technique for Program Planning - a Guide to Nominal Group and Delphi processes. Glenview, Illinois. Scott, Foresman and Company, 1975.

9. Proceedings "An international Consensus Conference to define core sets of outcome measures for Juvenile Systemic lupus Erythematosus and Juvenile Dermatomyositis" Pavia, Italy Almo Collegio Borromeo March 31-April 3, 2001.

10. Ruperto N, Ravelli A, Murray KJ, et al: For the Paediatric Rheumatology International Trials Organisation (PRINTO) and the Pediatric Rheumatology Collaborative Study Group (PRCSG) Preliminary Core Sets for disease damage assessment for Juvenile Systemic Lupus Erythematosus And Juvenile Dermatomyositis. Rheumatology 42: 1-8, 2003.

11. Tan EM, Cohen AS, Fries JF, et al: The 1982 revised criteria for the classification of Systemic Lupus Erythematosus. Arthritis Rheum 25: 1271-7, 1982
Sztajnbok, Universidade Estadual do Rio de Janeiro (UERJ); Blanca Elena Bica, Universidade Federal do Rio de Janeiro (UFRJ); Maria Odete Esteves Hilário, Universidade Federal de São Paulo (Unifesp); Iloite Scheibel, Universidade Federal do Rio Grande do Sul (UFRGS); Virginia Paes Leme Ferriani - Faculdade de Medicina de Ribeirão Preto/Universidade de São Paulo (FMRP-USP); Clovis Artur Almeida Silva - Faculdade de Medicina da Universidade de São Paulo (FMUSP); e Carlos Henrique Martins Silva - UFU-MG.

12. Hochberg MC: Updating the American College of Rheumatology revised criteria for the classification of Systemic Lupus Erythematosus. Arthritis Rheum 40: 1725-6, 1997.

13. Bohan A, Peter JB: Polymyositis and dermatomyositis. N Engl J Med 292:344-7, 1975.

14. Machado CSM, Ruperto N, Silva CHM, et al: For the Paediatric Rheumatology International Trials Organisation (PRINTO) The Brazilian version of the "Childhood Health Assessment Questionnaire - CHAQ" and the "Child Health Questionnaire - CHQ" Clin Exp Rheum (Suppl 23) 19: S25-30, 2001.

15. Brunner HI, Feldman BM, Bombardier C, Silverman ED: Sensitivity of the Systemic Lupus Erythematosus Disease Activity Index, British Isles Lupus Assessment Group Index, and Systemic Lupus Activity Measure in the evaluation of clinical change in childhood onset Systemic Lupus Erythematosus. Arthritis Rheum 42: 1354-60, 1999.

16. Brunner HI, Silverman ED, Bombardier C, Feldman B: European consensus Lupus activity measurement is sensitive to change in disease activity in childhood onset Systemic Lupus Erythematosus. Arthritis Care Res 49: 335-41, 2003.

17. Rider LG, Feldman BM, Perez MD, et al: Development and validated disease activity and damage indices for the juvenile idiopathic inflammatory myopathies. I- Physician, parent and patients global assessments. Arthritis Rheum 40:1976-83, 1997.

18. Lovell DJ, Lindsley CB, Rennenbohm RM, et al: Development and validated disease activity and damage indices for the juvenile idiopathic inflammatory myopathies - II. The Childhood Myositis Assessment Scale (CMAS): A quantitative tool for the evaluation of muscle function. Arthritis Rheum 42: 2213-9, 1999.

19. Huber AM, Feldman BM, Rennebohn RM, et al: Validation and clinical significance of the Childhood Myositis Assessment Scale (CMAS) for assessment in the Juvenile Idiopathic Myopathies. Arthritis Rheum 50: 1595- 603, 2004.

20. Huber AM, Hicks JE, Lachenbruch PA, et al: Validation of the Childhood Health Assessment Questionnaire in the Juvenile Idiopathic Inflammatory Myopathies. J Rheumatol 28: 1106-11, 2001.

21. Bode RK, Klein-Gitelman MS, Miller ML, Lechman TS, Pachman L: Disease activity score for children with juvenile dermatomyositis, reliability and validity evidence. Arthritis Rheum 49: 7-15, 2003.

22. Proceedings "International Consensus Conference to define core sets of outcome measures for Juvenile Systemic Lupus Erythematosus 
and Juvenile Dermatomyositis" Pavia, Italy - Almo Collegio Borromeo, March 31-April 3, 2001.

23. Smolen J, Strand V, Cardiel M, et al: Randomized clinical trials and longitudinal observational studies in systemic lupus erythematosus: consensus on a preliminary core set of outcome domains. J R heumatol 26: 504-7, 1999.

24. Miller FW, Rider LG, Chung Y-L, et al: For the International Myositis Outcome Assessment Collaborative Study Group Proposed preliminary core set measures for disease outcome assessment in adult and juvenile idiopathic inflammatory myopathies. Rheumatology 40: 1262-73, 2001

25. Rider L, Giannini EH, Harris-Love M, et al: For The International Myositis Assessment and Clinical Study (IMACS) Group - Defining clinical improvement in adult and juvenile myositis. J Rheumatol 30: 603-17, 2003.

26. Ruperto N, Buratti S, Duarte-Salazar C, et al: Health-related quality of life in juvenile-onset systemic lupus erythematosus and its relationship to disease activity and damage. Arthritis Care Res. 51:458-64, 2004.

27. Brunner EI, Giannini EH: Health related quality of life in children with rheumatic diseases. Curr Opin Rheumatol 15: 602-12, 2003.

28. Proceedings "An International Consensus Conference on Defining Improvement in Juvenile Systemic Lupus Erythematosus (JSLE) and Juvenile Dermatomyositis (JDM)- Camogli-Genoa-Italy September 27-30, 2003". 